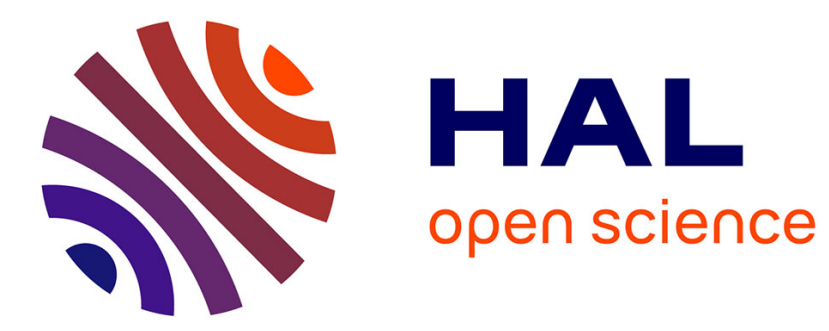

\title{
Impact de la sensibilité des contrôles hydrauliques sur les incertitudes hydrométriques
}

\author{
I. Horner, Jérôme Le Coz, Benjamin Renard, F. Branger
}

\section{To cite this version:}

I. Horner, Jérôme Le Coz, Benjamin Renard, F. Branger. Impact de la sensibilité des contrôles hydrauliques sur les incertitudes hydrométriques. La Houille Blanche - Revue internationale de l'eau, 2018, 1, pp.27-35. 10.1051/lhb/2018004 . hal-02608473

\section{HAL Id: hal-02608473 \\ https://hal.inrae.fr/hal-02608473}

Submitted on 16 May 2020

HAL is a multi-disciplinary open access archive for the deposit and dissemination of scientific research documents, whether they are published or not. The documents may come from teaching and research institutions in France or abroad, or from public or private research centers.
L'archive ouverte pluridisciplinaire HAL, est destinée au dépôt et à la diffusion de documents scientifiques de niveau recherche, publiés ou non, émanant des établissements d'enseignement et de recherche français ou étrangers, des laboratoires publics ou privés. 


\title{
Impact de la sensibilité des contrôles hydrauliques sur les incertitudes hydrométriques
}

\author{
Ivan HORNER, Jérôme LE COZ, Benjamin RENARD, Flora BRANGER
}

Irstea, UR RiverLy, centre de Lyon-Villeurbanne, 5 rue de la Doua, CS 20244, 69625 Villeurbanne, France

\begin{abstract}
RÉSUMÉ. - Les données hydrométriques sont affectées d'incertitudes dues aux erreurs de mesure des hauteurs d'eau, d'autant plus sévèrement que la sensibilité des contrôles hydrauliques de la station est faible. Dans un contexte tendant à la suppression ou à la modification des déversoirs hydrométriques, en lien avec la continuité écologique des cours d'eau, il est important de pouvoir quantifier de façon objective cette source d'incertitude. Une méthodologie générique s'appuyant sur l'approche bayésienne BaRatin est proposée et appliquée à cinq stations hydrométriques fictives présentant des déversoirs avec ou sans encoche, de sensibilité variable. Cet exercice confirme l'importance de la sensibilité du contrôle, en montrant qu'une encoche rectangulaire étroite, ou de préférence une encoche triangulaire, permet de contenir les incertitudes hydrométriques dans des gammes acceptables, en particulier pour les débits d'étiage.
\end{abstract}

Mots-clés : Hydrométrie ; erreur ; limnigramme ; incertitude ; débit ; sensibilité ; contrôle hydraulique ; BaRatin

\section{The impact of control sensitivity on hydrometric uncertainties}

\begin{abstract}
Hydrometric data are affected by uncertainties due to water level measurement errors, more severely as the sensitivity of the hydraulic controls of the station is low. In the context of the removal or modification of hydrometric weirs, in connection with the ecological continuity of streams, it is important to be able to objectively quantify this source of uncertainty. A general methodology based on the BaRatin Bayesian approach is proposed and applied to five fictitious hydrometric stations with weirs with or without notches, with variable sensitivity. This exercise confirms the importance of the sensitivity of the controls, showing that a narrow rectangular notch, and ideally a triangular notch, makes it possible to contain the hydrometric uncertainties within acceptable ranges, in particular for low flows.
\end{abstract}

Key-words: Hydrometry, error, stage series, uncertainty, discharge, sensitivity, hydraulic control, BaRatin

\section{INTRODUCTION}

Au niveau d'une station hydrométrique, un hydrogramme (série temporelle de débit d'un cours d'eau) est le plus souvent issu d'un limnigramme (série temporelle de hauteur d'eau) et d'une courbe de tarage (modèle hauteur-débit établi à partir d'un ensemble de mesures ponctuelles de hauteur $h$ et de débit $Q$ : les jaugeages). La relation hydraulique hauteur-débit est déterminée par les caractéristiques physiques du cours d'eau aux alentours de la station; on parle de contrôles hydrauliques. En particulier, à bas et moyen débit, la relation est le plus souvent contrôlée par un radier naturel ou un seuil artificiel ou déversoir situé à l'aval de la station.

Certains contrôles artificiels, en plus d'offrir une meilleure stabilité dans le temps, ont des géométries permettant une plus grande sensibilité, ce qui permet de réduire les incertitudes liées aux erreurs limnimétriques. Pour une variation de débit donnée, un contrôle est d'autant plus sensible que la variation de hauteur correspondante est grande, c'est-à-dire que le rapport $\mathrm{d} h / \mathrm{d} Q$ est grand. Cependant, les contrôles artificiels doivent de plus en plus souvent être détruits ou adaptés pour améliorer la continuité écologique des cours d'eau, sans que l'impact sur les incertitudes associées aux hydrogrammes ne soit objectivement quantifié.

La propagation des incertitudes limnimétriques sur les hydrogrammes est prise en compte dans les méthodes traditionnelles d'analyse d'incertitude, en général fondées sur l'analyse des résidus de régression liénaire (Dymond et Christian, 1982 ; Herschy, 1999; Petersen-Overleir et Reitan, 2005 ; WMO, 2006; Olivier et al., 2008 ; ISO 1100, 2010). Cependant ces méthodes restent très exceptionnellement appliquées par les services hydrométriques opérationnels, et elles ne fournissent pas de résultats probabilistes (sous la forme d'échantillons) facilement manipulables, mais en général des écart-types ou incertitudes-types. D'autre part, si l'importance des erreurs limnimétriques systématiques (ou corrélées) a été reconnue par Petersen-Overleir et Reitan (2005), ils ne les ont pas considérées dans leurs calculs, comme c'est aussi le cas des autres méthodes publiées. Or les erreurs limnimétriques systématiques ne sont pas réduites par l'agrégation temporelle des débits moyens, caractéristiques du régime hydrologique moyen ou d'étiage.

Une méthode originale (cf. Horner et al., soumis) pour prendre en compte les erreurs limnimétriques et les propager aux hydrogrammes a été développée, testée et implémentée dans la nouvelle version du logiciel BaRatinAGE, qui permet le déploiement opérationnel de la méthode BaRatin introduite par Le Coz et al. (2013, 2014). Cette méthode propage deux types d'erreurs limnimétriques: les erreurs non-systématiques (bruit instrumental, vaguelettes...) et systématiques (erreurs d'étalonnage, de calage et de représentativité de l'écoulement moyen). Cette méthode est ici appliquée à cinq stations fictives munies de déversoirs à différentes sensibilités, avec ou sans encoche pour contenir 
90\% des débits les plus bas. Les données synthétiques (limnigramme, jaugeages, courbes de tarage) ont été générées à partir d'un hydrogramme réel (l'Yzeron à Craponne) de façon à conserver le maximum de paramètres comparables. N.B. Dans le cadre de cette étude, seules les incertitudes limnimétriques sont étudiées car ce sont sur elles que la sensibilité des contrôles hydrauliques a un impact. Les incertitudes des hydrogrammes dues aux incertitudes paramétriques et structurelles de la courbe de tarage sont également propagées mais ne sont pas considérées ici.

\section{MÉTHODE ET EXPÉRIENCE SUR DONNÉES SYNTHÉTIQUES}

\section{II.1. Principe général de l'approche}

Nous avons créé plusieurs stations hydrométriques fictives où seuls les contrôles hydrauliques (en particulier leurs sensibilités) sont différents, toutes choses étant égales (ou équivalentes) par ailleurs. Ces stations hydrométriques fictives suivent la même chronique de débit, et donc le même régime hydrologique, issue d'un même bassin versant réel. La méthode se décompose en plusieurs étapes succinctement présentées ci-dessous et décrites plus en détails dans la suite du document.

— Définition d'un hydrogramme $Q_{t h}(t)$ commun à toutes les stations, instantané à pas de temps variable ; cette série temporelle de débit est issue d'une station hydrométrique réelle, dont elle représente le régime hydrologique sur les années d'enregistrement considérées.

- Définition des contrôles hydrauliques des stations fictives à tester : on définit la géométrie des contrôles de la station $i$ ( $i=1 \ldots 5$, pour chacune des 5 stations hydrométriques fictives retenues pour l'étude) à partir de laquelle une équation théorique $Q=f_{i, t h}(h)$ de la courbe de tarage est définie.

- Création des limnigrammes synthétiques avec incertitudes : une série temporelle de hauteur $h_{i}(t)$ est créée pour chacune des stations $i$ à partir de l'équation de la courbe de tarage inversée $h=f_{i, t h}{ }^{-1}(Q)$; les erreurs limnimétriques systématiques et non-systématiques sont échantillonnées selon des distributions gaussiennes de moyenne nulle et d'écart-type correspondant à leur incertitude-type supposée. Les erreurs non-systématiques (batillage, bruit instrumental, vaguelettes...) sont ré-échantillonnées à chaque pas de temps tandis que les erreurs systématiques (calage et dérive du capteur par rapport à l'échelle limnimétrique de référence) ne sont ré-échantillonnées que périodiquement, en lien avec les recalages du capteur lors des visites de contrôle. L'échantillonnage de ces erreurs permet de créer, pour chaque station $i, N$ réalisations du limnigramme $h_{i}(t)$ rendant ainsi compte des incertitudes limnimétriques.

- Application de la méthode BaRatin : des aprioris hydrauliques larges sont définis à partir des géométries des différentes stations, et des jaugeages synthétiques sont créés en simulant leur dispersion autour de la courbe de tarage théorique. BaRatin permet alors d'estimer une courbe de tarage et un hydrogramme avec leurs incertitudes, en propageant les incertitudes, paramétriques, structurelles et limnimétriques selon la méthode introduite par Horner et al. (soumis) et détaillée dans la documentation de BaRatinAGE 2.1 (2016). BaRatin produit $N$ réalisations de l'hydrogramme permettant de calculer l'incertitude associée à celui-ci (e.g. calcul de l'enveloppe d'incertitude à $95 \%$ par le calcul des quantiles $2.5 \%$ et $97.5 \%$ ).
- Post-traitement de l'hydrogramme et de ses incertitudes : on ne conserve pour cette étude que les incertitudes limnimétriques, avant agrégation à différents pas de temps (débits moyens horaires, journaliers, mensuels, annuels...) et calcul de moyennes mobiles comme le VCN30, minimum annuel des moyennes mobiles sur 30 jours consécutifs. Ces différents calculs sont réalisés sur chacune des $N$ réalisations de l'hydrogramme. Les incertitudes associées peuvent ensuite être obtenues à partir des $N$ résultats de ces calculs.

\section{II.2. Génération des données synthétiques}

L'hydrogramme théorique choisi est l'hydrogramme officiel de la banque Hydro pour la station de l'Yzeron à Craponne (Code station : V3015010 ; Producteur : DREAL Auvergne-Rhône-Alpes). Les coordonnées du point de mesure en LAMBERT II étendu sont $(785470 ; 2084490)$ et l'altitude du zéro de l'échelle vaut 234.22 m NGF IGN69. La station, mise en service le 26/10/1969, suit un bassin versant péri-urbain de $48 \mathrm{~km}^{2}$ situé dans l'Ouest lyonnais. Cette station a été choisie pour la longue chronique disponible et l'absence de lacune. L'Yzeron est typique de cours d'eau petits à moyens dont la station hydrométrique est susceptible d'être équipée d'un seuil maçonné.

L'hydrologie du bassin versant est influencée par différents usages mais sans dispositif de soutien d'étiage; elle se caractérise par des crues rapides importantes et de longues périodes d'étiage. Voici quelques débits caractéristiques : Module : $0,325 \mathrm{~m}^{3} /$; VCN10 : $0,007 \mathrm{~m}^{3} / \mathrm{s}$; Débit maximum enregistré : $54 \mathrm{~m}^{3} / \mathrm{s}$. La courbe des débits classés montre que $95 \%$ des débits moyens journaliers sont compris entre $0,01 \mathrm{~m}^{3} / \mathrm{s}$ et $1 \mathrm{~m}^{3} / \mathrm{s}$; toujours en moyennes journalières, le débit non dépassé pour $50 \%$ des pas de temps est $0,172 \mathrm{~m}^{3} / \mathrm{s}$ et le débit non dépassé pour $90 \%$ des pas de temps est $0,82 \mathrm{~m}^{3} / \mathrm{s}$.

C'est cette dernière valeur qui a servi à dimensionner des scénarios d'encoches ménagées dans un seuil rectangulaire initial. Les 5 géométries présentées sur la Figure 1 ont été considérées. La première station fictive est supposée munie d'un déversoir à crête horizontale de chute et largeur réalistes par rapport au cours d'eau réel. Les quatre autres stations fictives ont des déversoirs qui combinent ce déversoir rectangulaire initial avec une encoche rectangulaire plus ou moins large $(1 \mathrm{~m}$ ou $3 \mathrm{~m})$ ou une encoche triangulaire plus ou moins évasée $\left(90^{\circ}\right.$ ou $\left.120^{\circ}\right)$. Les encoches ont été dimensionnées pour contenir à peu près le débit dont la valeur correspond au quantile à $90 \%$ des débits moyens journaliers, soit un débit de débordement de l'encoche d'environ $0,8 \mathrm{~m}^{3} / \mathrm{s}$. Les dimensions des encoches obtenues sont jugées réalistes.

Les équations et paramètres des courbes de tarage théoriques de ces cinq stations fictives sont fournis en Annexe 1, et les courbes de tarage sont présentées sur la Figure 2. Afin de visualiser les différences de sensibilité des différentes stations, il est possible, pour une gamme de débit donnée (ici, de $1 \mathrm{~L} / \mathrm{s}$ à $50 \mathrm{~m}^{3} / \mathrm{s}$ ) de calculer la sensibilité $s$ suivant la formule suivante :

$$
s=\frac{Q d h}{d Q}
$$

Où $Q$ est le débit et $d h$ et $d Q$ sont respectivement une variation de hauteur d'eau et la variation de débit correspondante. Avec cette expression, la sensibilité $s$ est définie par rapport à une variation relative du débit (et non absolue comme dans l'introduction); elle a la dimension d'une longueur et 


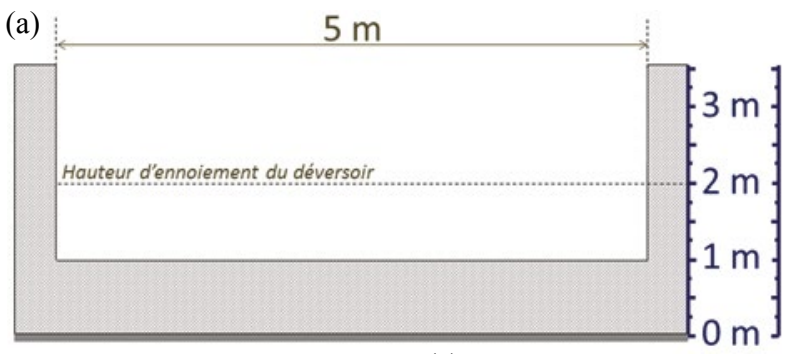

(b)

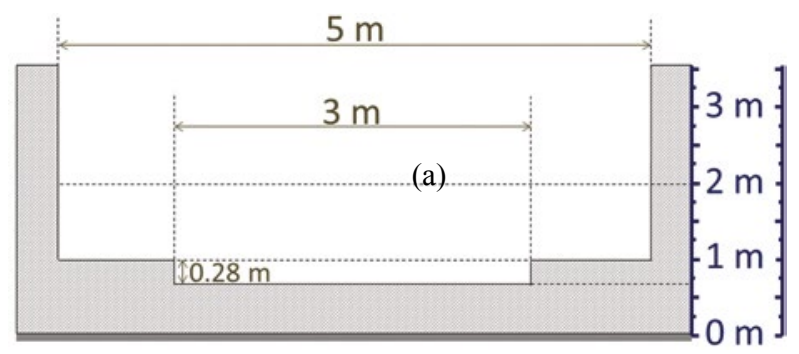

(d)

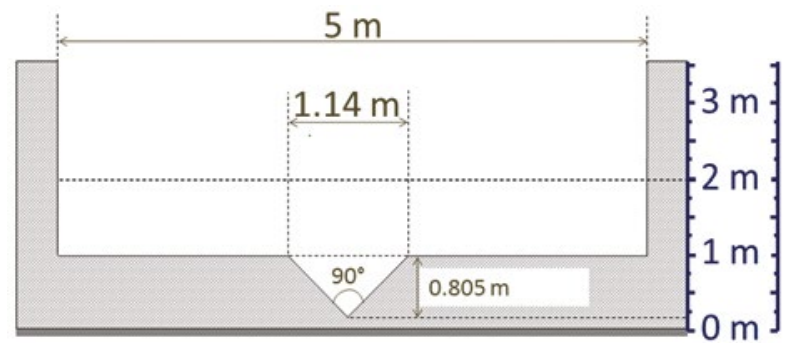

(c)

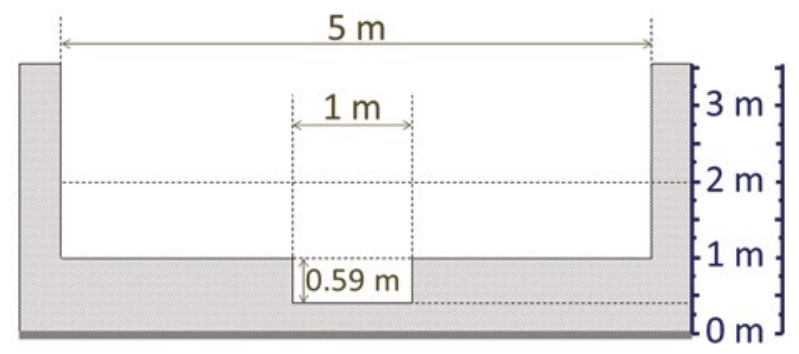

(e)

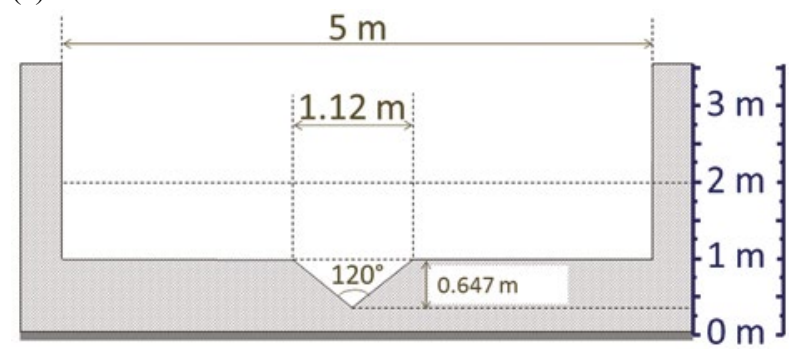

Figure 1 : Caractéristiques géométriques des déversoirs des cinq stations hydrométriques fictives étudiées : seuil rectangulaire initial (a), avec encoche rectangulaire de $3 \mathrm{~m}$ (b) ou de $1 \mathrm{~m}$ (c), avec encoche triangulaire de $90^{\circ}$ (d) ou de $120^{\circ}$ (e).

indique, pour un débit donné, la variation de hauteur d'eau en mètres correspondant à une variation relative du débit de $100 \%$ (la même valeur peut se lire en centimètres pour une variation relative du débit de $1 \%$ ). Plus $s$ est grand, plus la sensibilité est grande, et vice versa. Sur la Figure 3, on vérifie que les déversoirs triangulaires présentent une décroissance moins rapide de la sensibilité pour les faibles débits, la pente de la relation étant gouvernée par l'exposant de l'équation du contrôle. Cependant, la sensibilité dépend aussi et surtout de la largeur au miroir de la section de contrôle, ce qui explique que le déversoir rectangulaire étroit (largeur
$1 \mathrm{~m})$ puisse devenir plus sensible que les déversoirs triangulaires pour les débits supérieurs à 0,2 et $0,3 \mathrm{~m}^{3} / \mathrm{s}$. Lorsque l'encoche déborde, la sensibilité chute quel que soit le scénario pour se rapprocher de la sensibilité du déversoir rectangulaire initial, alors activé.

Les limnigrammes sont alors calculés à partir de l'inverse des courbes de tarage de chaque station fictive. Pour des raisons pratiques, un barème, très fin (environ 34000 valeurs), a été utilisé. Pour les débits nuls, la valeur du limnigramme a été prise égale aux zéros hydrauliques théoriques de chaque station. Pour échantillonner les erreurs limnimétriques

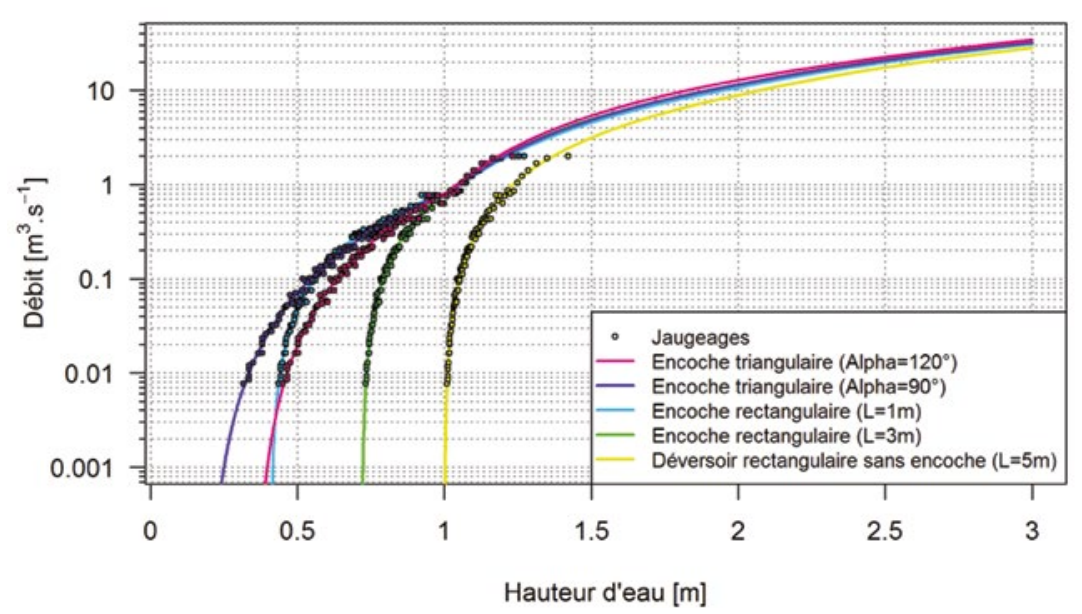

Figure 2 : Courbes de tarage théoriques et jaugeages synthétiques des cinq stations hydrométriques fictives. 


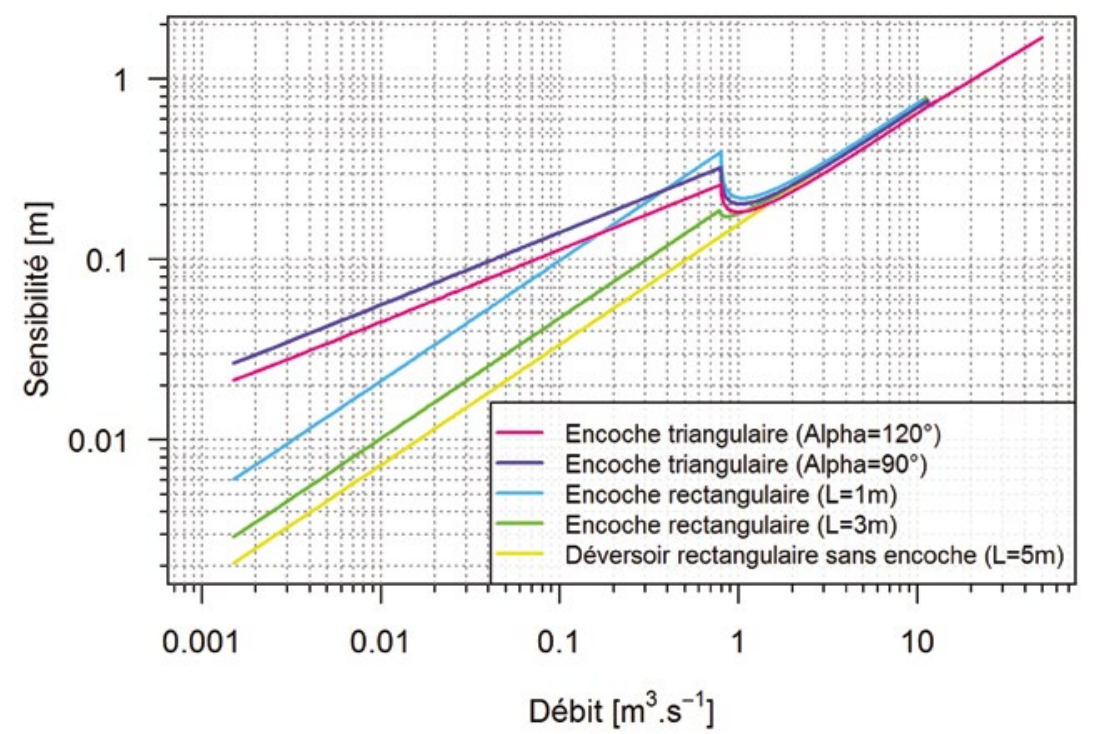

Figure 3 : Sensibilité des cinq stations hydrométriques fictives (cf. Equation 1).

systématiques et non-systématiques, la même valeur d'incertitude-type a été supposée : $0,005 \mathrm{~m}$ soit $\pm 1 \mathrm{~cm}$ d'incertitude élargie à $95 \%$. Cette incertitude est arbitraire mais elle semble réaliste pour les deux sources d'erreur. Au vu des pratiques courantes en termes de vérification et recalage du capteur limnimétrique, un ré-échantillonnage périodique de l'erreur limnimétrique systématique tous les 30 jours a été retenu. Bien entendu, chaque cas réel doit idéalement faire l'objet d'une analyse appropriée des incertitudes de mesure des hauteurs d'eau. Dans le cas présent, les erreurs systématiques sont constantes entre deux ré-échantillonnages. La variation de ces erreurs pourrait suivre une dynamique plus complexe (par exemple une évolution linéaire entre les deux recalages pour rendre compte de façon plus réaliste de la dérive du capteur, cf. Horner et al., soumis). L'approximation faite paraît raisonnable au vu de notre compréhension très superficielle des erreurs liées aux dérives des capteurs.

Les débits théoriques $Q_{j, t h}$ puis les hauteurs d'eau $h_{j, i}$ correspondantes de chaque jaugeage $j$ sont calculés à partir de la courbe des débits classés à pas de temps horaires et d'une séquence de 50 probabilités de non dépassement également réparties entre 0.03 et 0.99 . Afin de simuler la dispersion des jaugeages autour de la courbe, due aux incertitudes de mesure et aux incertitudes structurelles du modèle de courbe de tarage, une erreur relative $e_{j}$ est échantillonnée dans une distribution gaussienne de moyenne nulle et d'écart-type 0.1 puis ajoutée à chaque débit jaugé théorique $Q_{j, t h}$ de la façon suivante : $Q_{j}=Q_{j, t h} \times\left(1+e_{j}\right)$. Pour chaque courbe de tarage $i$, on dispose ainsi d'un jeu de 50 jaugeages $\left(Q_{j}, h_{j, i}\right)_{j=1,2, \ldots, 50}$ (cf. Figure 2).

\section{II.3. Estimation des courbes de tarage et des hydrogrammes avec BaRatin}

Les aprioris nécessaires à BaRatin (cf. Annexe 2) sont définis à partir des géométries des différentes stations présentées plus haut et des courbes de tarage théoriques données en Annexe 1. L'équation de la courbe de tarage BaRatin peut différer légèrement de l'équation de la courbe de tarage théorique, afin de respecter le cadre général de l'équation des contrôles de BaRatin (cf ; Le Coz et al., 2014), et éviter les coefficients négatifs (interdits). Les valeurs centrales des paramètres $k$ (hauteurs d'activation), $a$ et $c$ sont calculées directement à partir des géométries des contrôles. Des valeurs d'incertitudes arbitraires sont choisies pour les paramètres de chaque loi puissance. Pour rappel, dans le cadre de cette étude, toutes les composantes d'incertitude (paramétrique, structurelle et limnimétriques) sont propagées sur les hydrogrammes, mais seules les incertitudes limnimétriques sont examinées car ce sont sur elles que la sensibilité des contrôles hydrauliques a un impact.

Dans le cas 0 (Déversoir rectangulaire sans encoche), le contrôle de section laisse la place à un contrôle par le chenal, lorsque le déversoir est noyé. Pour les quatre autres configurations, la même transition s'opère, mais le déversoir est représenté par deux segments : à bas débit, seule l'encoche est active, puis le reste du déversoir rectangulaire initial s'ajoute à l'encoche. A noter que l'expansion latérale de la surface mouillée des encoches triangulaires après l'activation du déversoir rectangulaire initial (à la cote $1 \mathrm{~m}$ ) est négligée ici, alors qu'elle était retranchée dans les équations des courbes de tarage théoriques. Les jaugeages synthétiques permettront de corriger les paramètres du déversoir rectangulaire lors de l'estimation des courbes de tarage par BaRatin. Dans tous les cas, les résultats se concentreront sur les incertitudes provenant du limnigramme et non de la courbe de tarage. Une incertitude élargie de mesure de $\pm 10 \%$ est assignée à chaque jaugeage. Les courbes de tarage estimées par BaRatin sont en accord étroit avec les jaugeages et les courbes théoriques, avec des incertitudes acceptables. Les distributions a posteriori obtenues pour les différents paramètres des courbes de tarage sont réalistes et compatibles avec les distributions a priori.

\section{RÉSULTATS}

Dans les paragraphes suivants, on présente de la même façon les résultats obtenus pour les débits moyens horaires, mensuels et annuels, ainsi que le VCN30. Sur chaque figure, à gauche, l'hydrogramme du cas 0 agrégé à pas de temps horaire est présenté, ainsi que les incertitudes limnimétriques, c.à.d. la demi-longueur de l'intervalle d'incertitude à 
$95 \%$ divisé par le débit de la courbe de tarage calculée par BaRatin (« MaxPost»), pour chacune des stations hydrométriques fictives, en respectant toujours le même code couleur. A droite, les incertitudes limnimétriques totales (systématiques et non systématiques combinées) sont mises en regard des débits classés sur la période considérée, au pas de temps d'agrégation considéré.

\section{III.1. Incertitude des débits moyens horaires}

L'évaluation des incertitudes limnimétriques des débits moyens horaires sur l'année 2010 (Figure 4) montre un très fort impact de la sensibilité des contrôles sur les débits d'étiage, tandis que les débits de crue ont toujours des incertitudes faibles. L'incertitude relative peut atteindre des valeurs extrêmement fortes en situation d'étiage sévère, notamment avant le pic de crue de septembre 2010, quand les débits deviennent très faibles. Pour le déversoir rectangulaire sans encoche, l'incertitude limnimétrique dépasse les $300 \%$ et les encoches rectangulaires ne parviennent pas à la réduire à des valeurs acceptables $(200 \%$ et $80 \%$ environ). Seules les encoches triangulaires permettent de contenir cette composante d'incertitude (qui n'est pas la seule à affecter le débit !) sous les $25 \%$, voire $10 \%$ la plupart du temps.

\section{III.2. Incertitude des débits moyens mensuels}

Les incertitudes limnimétriques des débits moyens mensuels sur la période 2000-2009 (Figure 5) sont plus faibles, car les erreurs non systématiques sont rapidement réduites par l'agrégation temporelle (cf. Horner et al., soumis). Cependant, les mêmes tendances se dégagent, avec des incertitudes toujours très fortes (jusqu'à environ $100 \%$ ) pour les mois secs avec le déversoir rectangulaire sans encoche, et peu d'amélioration avec l'encoche rectangulaire large. L'encoche rectangulaire étroite permet de limiter les incertitudes limnimétriques à $30 \%$ environ, mais à nouveau, ce sont les encoches triangulaires qui permettent véritablement de maîtriser des incertitudes acceptables (inférieures à $15 \%$ ou $10 \%$ ) même lors des mois les plus secs.

\section{III.3. Incertitude des débits moyens annuels}

A nouveau, les incertitudes limnimétriques des débits moyens annuels sur la période 1970-2009 (Figure 6) présentent les mêmes tendances, à ceci près que les incertitudes sont beaucoup plus petites, de $1 \%$ à $5 \%$ pour les bas débits et le contrôle le moins sensible. On note également que le déversoir avec encoche rectangulaire étroite produit cette fois-ci des incertitudes plus faibles que le déversoir avec
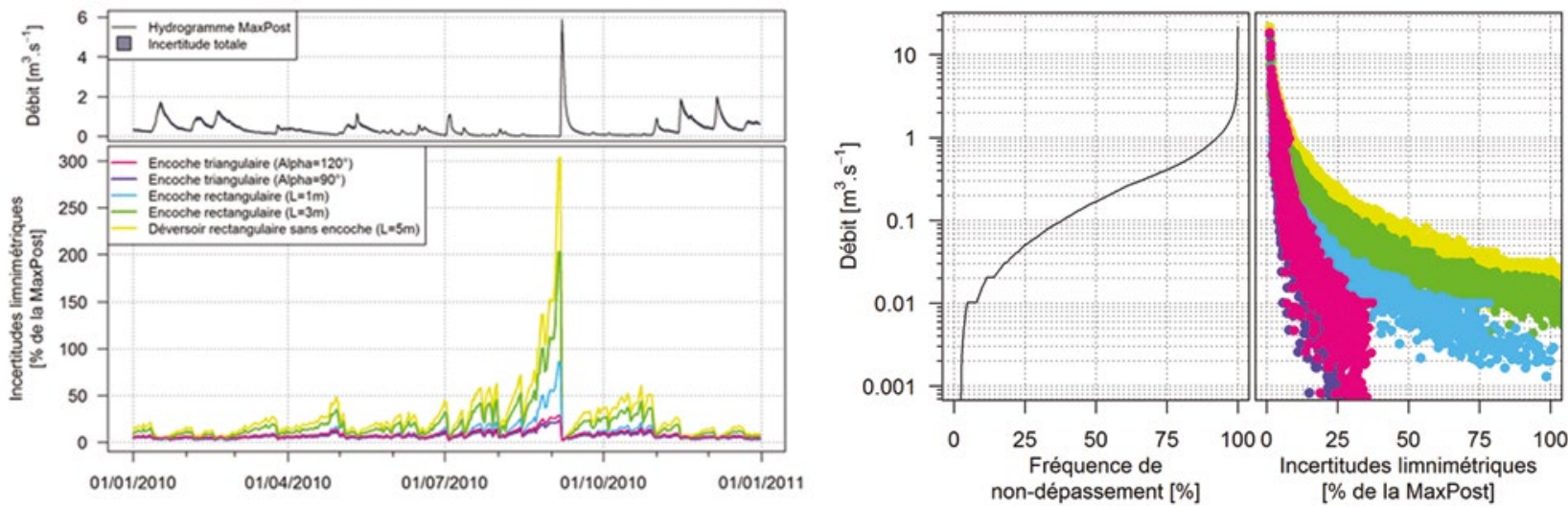

Figure 4 : Incertitudes limnimétriques des débits moyens horaires sur l'année 2010, au cours du temps (à gauche) et en fonction des débits classés (à droite) pour les cinq configurations de stations hydrométriques fictives. Les incertitudes relatives sont exprimées en pourcentage du débit issu de la courbe de tarage calculée par BaRatin (" MaxPost »).
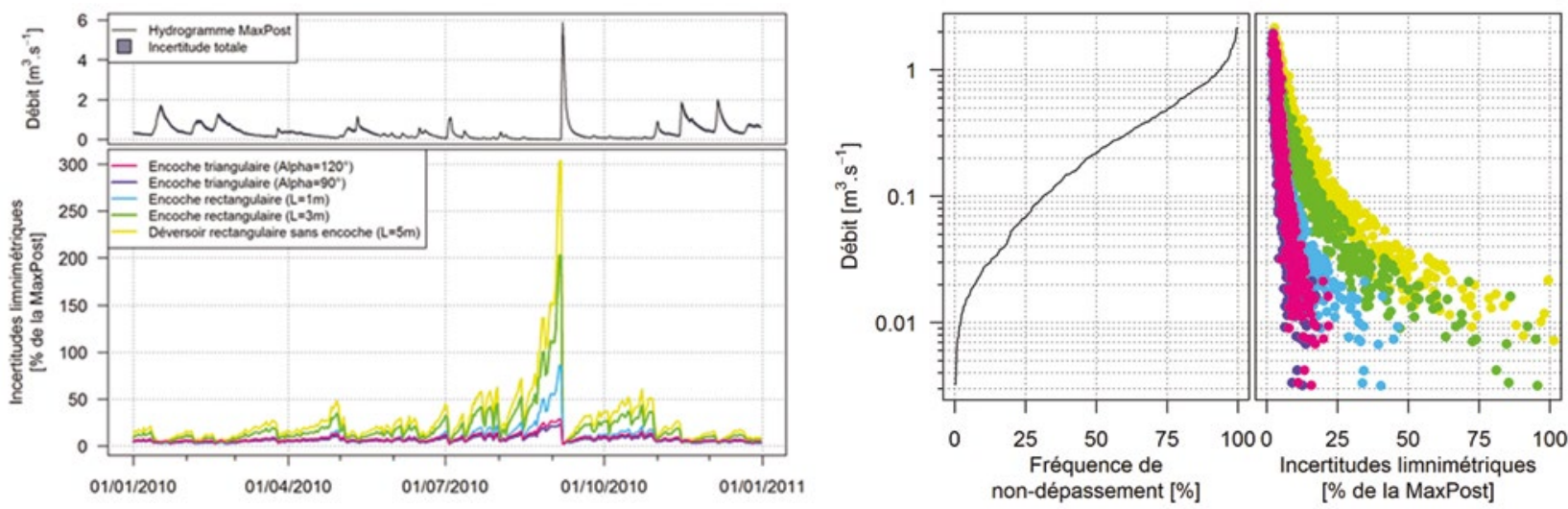

Figure 5 : Incertitudes limnimétriques des débits moyens mensuels sur la période 2000-2009, au cours du temps (à gauche) et en fonction des débits classés (à droite) pour les cinq configurations de stations hydrométriques fictives. 

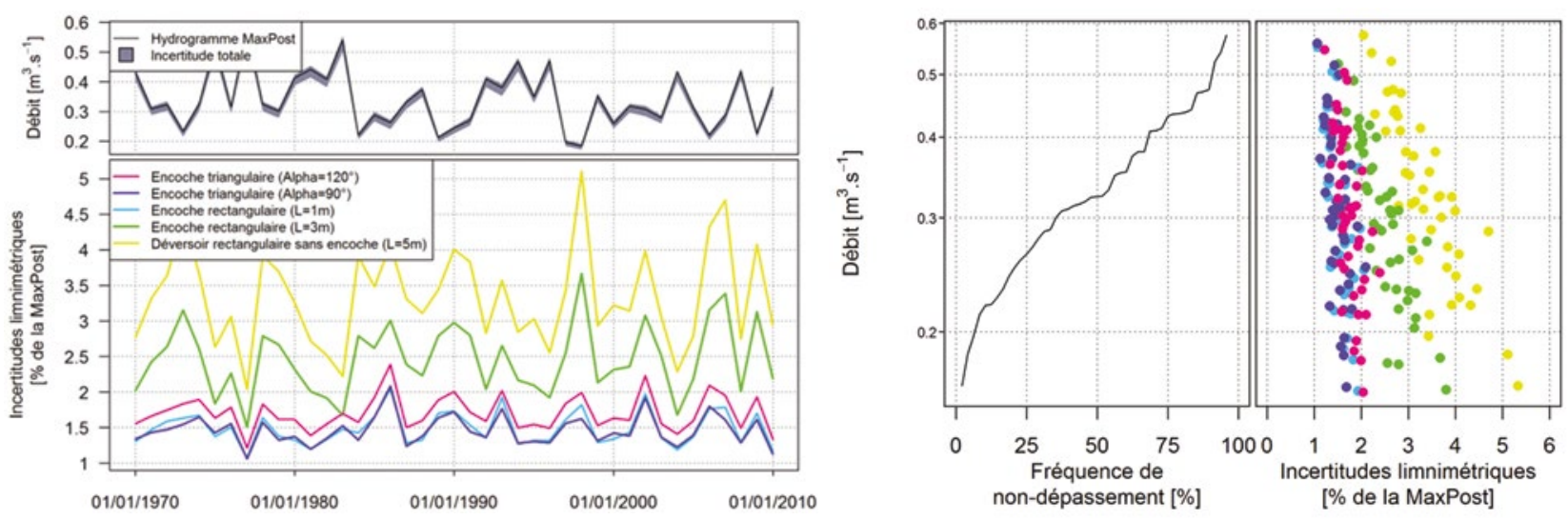

Figure 6 : Incertitudes limnimétriques des débits moyens annuels sur la période 1970-2009, au cours du temps (à gauche) et en fonction des débits classés (à droite) pour les cinq configurations de stations hydrométriques fictives. Notez le changement de l'échelle des incertitudes à droite.

encoche triangulaire évasée $\left(120^{\circ}\right)$, et équivalentes à celles du déversoir avec encoche triangulaire à $90^{\circ}$. Ceci peut s'expliquer par la sensibilité supérieure de l'encoche rectangulaire étroite pour la gamme des débits intermédiaires, entre les plus faibles et le débordement, comme déjà vu sur la Figure 3. Par construction, les encoches sont toutes dimensionnées pour contenir le quantile $90 \%$ des débits moyens journaliers, et il est probable que la gamme des débits intermédiaires soit prépondérante dans la moyenne annuelle, d'où la meilleure performance de l'encoche rectangulaire étroite. Ceci illustre que les incertitudes limnimétriques ne dépendent pas seulement des incertitudes de mesure des hauteurs d'eau et de la sensibilité des contrôles, mais aussi du régime hydrologique et du pas de temps d'agrégation.

\section{III.4. Incertitude des VCN30 (indicateur d'étiage)}

Enfin, nous nous intéressons aux incertitudes limnimétriques des VCN sur 30 jours sur la période 1970-2009 (Figure 7). Par définition, le VCN30 est le minimum annuel de la moyenne mobile calculée sur 30 jours consécutifs. C'est un indicateur hydrologique couramment employé pour caractériser la situation d'étiage de l'année en cours. Comme attendu, les écarts d'incertitude entre stations fictives sont encore plus prononcés, avec des incertitudes extrêmement fortes sur les VCN30 les plus faibles et ramenées à des valeurs inférieures à $20 \%$ par l'emploi d'encoches triangulaires, les encoches rectangulaires restant trop peu sensibles pour les débits d'étiage les plus bas.

\section{CONCLUSION}

S'appuyant sur la méthode bayésienne BaRatin (Le Coz et al., 2013, 2014 ; Horner et al., soumis), la méthodologie proposée dans cet article permet de propager les incertitudes limnimétriques vers les hydrogrammes et leurs moyennes à différents pas de temps, et in fine vers les statistiques hydrologiques. Ceci permet de quantifier l'impact de la sensibilité des contrôles d'une station hydrométrique donnée sur les données hydrologiques. La méthode tient compte de deux composantes d'incertitude limnimétrique, l'une due aux erreurs non systématiques (batillage, bruit instrumental...), l'autre due aux erreurs systématiques (calage et dérive du capteur par rapport à l'échelle, avec ré-échantillonnage périodique de l'erreur lors des recalages de la station).

L'exercice théorique présenté ici a été construit à partir de l'hydrologie du cas réel de l'Yzeron à Craponne, jugé représentatif d'un cours d'eau à étiages prononcés et de taille modeste équipé d'un seuil hydrométrique susceptible
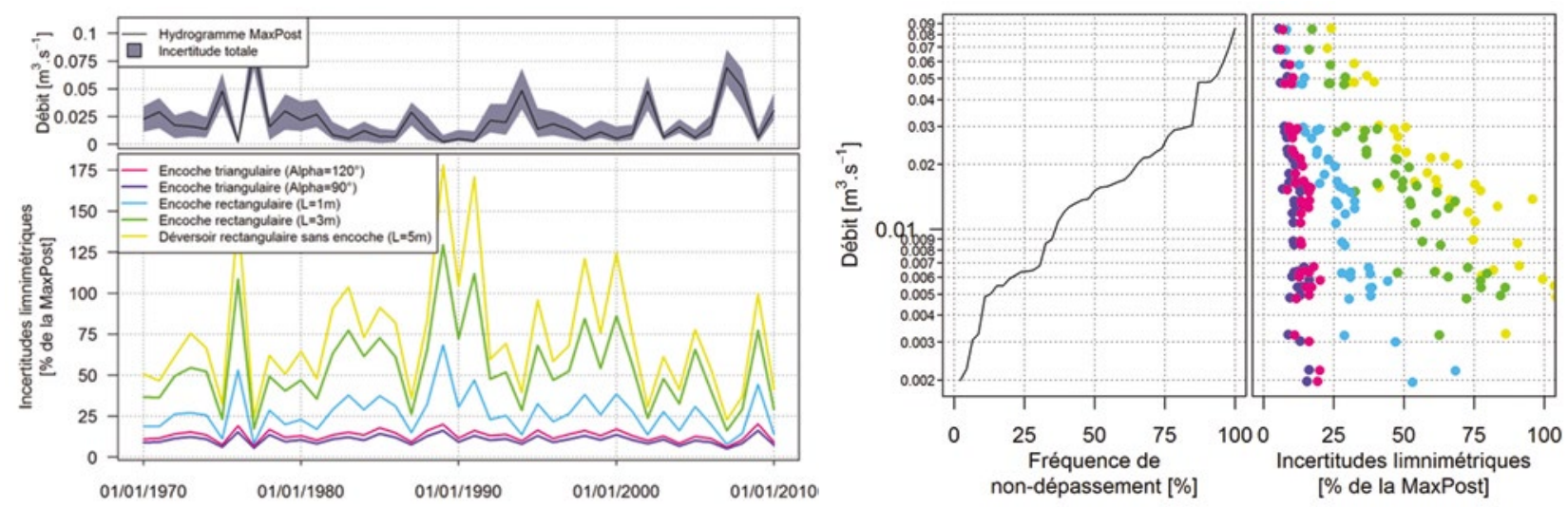

Figure 7 : Incertitudes limnimétriques des VCN sur 30 jours sur la période 1970-2009, au cours du temps (à gauche) et en fonction des débits classés (à droite) pour les cinq configurations de stations hydrométriques fictives. 
d'être modifié. Des données synthétiques (hauteurs d'eau, jaugeages, courbes de tarage) avec incertitudes ont été générées pour cinq configurations réalistes de déversoirs allant d'un simple seuil horizontal au même seuil muni d'une encoche rectangulaire plus ou moins large (1 ou $3 \mathrm{~m}$ ) ou d'une encoche triangulaire plus ou moins évasée $\left(90^{\circ}\right.$ ou $120^{\circ}$ ). Chaque encoche a été dimensionnée de façon à contenir le débit dont la valeur correspond au quantile à $90 \%$ des débits moyens journaliers.

L'impact d'une encoche rectangulaire étroite, et idéalement triangulaire, sur la sensibilité de la courbe de tarage est très important, ce qui permet de réduire fortement les incertitudes sur les hydrogrammes produits, même aux pas de temps mensuels et annuels, malgré la réduction des erreurs limnimétriques non systématiques par l'agrégation temporelle. La réduction d'incertitude est surtout sensible pour les débits faibles à moyens, passant dans l'encoche ; elle est particulièrement importante pour les débits caractéristiques de l'étiage, comme le montre l'étude du VCN30.

Il est évident que les incertitudes relatives (en \% du débit) croissent inévitablement quand le débit tend vers zéro. Cependant, l'impact important qui subsiste sur les moyennes mensuelles et annuelles montre qu'il ne s'agit pas de valeurs singulières sans représentativité à l'échelle du régime hydrologique. Par ailleurs, même si les incertitudes limnimétriques sont contenues, la mesure des faibles débits peut aussi être compromise par les incertitudes paramétrique et structurelle de la courbe de tarage. On s'attend néanmoins à une incertitude structurelle modérée pour un déversoir idéal dont l'équation hydraulique est bien connue. La composante paramétrique sera également modérée si l'on dispose de suffisamment de jaugeages à bas débit, dont les incertitudes de mesure ne doivent pas être sous-estimées.

Les résultats de cette étude qui se focalise uniquement sur les incertitudes limnimétriques auraient pu être obtenus directement en utilisant les équations théoriques des courbes de tarage. Il nous est apparu plus pertinent de proposer une méthode complète pouvant s'appliquer opérationnellement à n'importe quel cas réel de station hydrométrique. De plus, l'utilisation de la méthode BaRatin permet potentiellement de ne pas se limiter aux incertitudes limnimétriques et de considérer les incertitudes de la courbe de tarage, en vue de bilans complets de toutes les composantes d'incertitude.

On dispose donc d'une méthode quantitative générique pour l'analyse des incertitudes limnimétriques et de courbe de tarage applicable à chaque cas (station réelle ou scénario d'aménagement) à l'aide du logiciel opérationnel BaRatinAGE. De tels chiffres sont susceptibles d'objectiver l'intérêt de maintenir et transformer les seuils hydrométriques existants, voire d'en construire de nouveaux, si l'on veut disposer de données hydrologiques d'incertitudes acceptables, surtout pour les étiages, ce qui est crucial pour la gestion durable des ressources en eau et des milieux aquatiques. Plutôt que de détruire ou araser les seuils hydrométriques, ce qui aboutirait à des sensibilités catastrophiques en situation d'étiage (sans parler des problèmes d'instabilité de la relation hauteur-débit, ou détarages), il convient donc d'étudier des designs alternatifs, ménageant une encoche pour les bas débits, afin de concilier précision hydrométrique et franchissabilité par les espèces à enjeu sur le secteur de la station. Il reste naturellement utile de chercher aussi à réduire les incertitudes de mesure des enregistrements de hauteur d'eau et à favoriser la stabilité des contrôles au cours du temps.

\section{REMERCIEMENTS}

Le développement de la méthode BaRatin depuis 2010 est soutenu par Irstea, le SCHAPI (services hydrologiques nationaux français), et la Compagnie nationale du Rhône (CNR). La méthode de propagation des incertitudes limnimétriques et la présente étude ont été produites par I. Horner dans le cadre de contrats de recherche avec la CNR et le SCHAPI (convention SRNH DGPR) en 2015 et 2016. Le projet FloodScale (2012-2015) est soutenu par l'Agence Nationale de la Recherche (ANR) contrat $\mathrm{n}^{\circ}$ ANR 2011 BS56 027 et contribue au programme HyMeX. Le projet PHC Dumont D'Urville 34185SH (2015-2016) est financé par le ministère des Affaires étrangères et européennes et le ministère de l'Enseignement supérieur et de la Recherche. BaRatin utilise la bibliothèque Fortran DMSL développée par Dmitri Kavetski de l'Université d'Adélaïde, en Australie.

\section{RÉFÉRENCES}

Logiciel BaRatinAGE v2.1 (2016) - Aide.

DYMond J.R., Christian, R. (1982) - Accuracy of discharge determined from a rating curve. Hydrological Sciences Journal/ Journal des Sciences Hydrologiques, 27(4), 493-504.

Herschy, R.W. (1999) - Hydrometry, Principles and Practices, Wiley.

Horner I., Renard B., Le Coz J., Branger F., McMillan H.K., PierRefeu G. Impact of stage measurement errors on streamflow uncertainty (submitted). Water Resources Research.

ISO 1100-2:2010 (2010) - Hydrometry - Measurement of liquid flow in open channels. Part 2: Determination of the stage-discharge relationship. International Organization for Standardization, Genève, Suisse, 28 p.

Le Coz, J., Chaléon, C., Bonnifait, L., Branger, B. Le Boursicaud, R., Renard, B. Branger, F., Diribarne, J., Valente, M. (2013) - Analyse bayésienne des courbes de tarage et de leurs incertitudes : la méthode BaRatin. La Houille Blanche, 6, 31-41.

Le Coz, J., Renard, B., Bonnifait, L., Branger, F., LE Boursicaud, R. (2014) - Combining hydraulic knowledge and uncertain gaugings in the estimation of hydrometric rating curves: a Bayesian approach. Journal of Hydrology, 509, $573-587$

Olivier, A., Pierrefeu, G., Scotti, M., Blanquart, B. (2008) - Incertitudes sur les débits issus des courbes de tarage. Conférence SHF « Mesures hydrologiques et incertitudes 》.

Petersen-Overleir, A., Reitan, T. (2005) - Uncertainty in flood discharges from urban and small rural catchments due to inaccurate head measurements. Nordic Hydrology, 36(3), 245-257.

World Meteorological Organization (2006) - Technical regulations, Hydrology. WMO-No. 49, Vol. III, 112 p. 


\section{ANNEXES}

Annexe 1. Equations et paramètres des courbes de tarage théoriques des cinq stations hydrométriques fictives étudiées dans cet article.

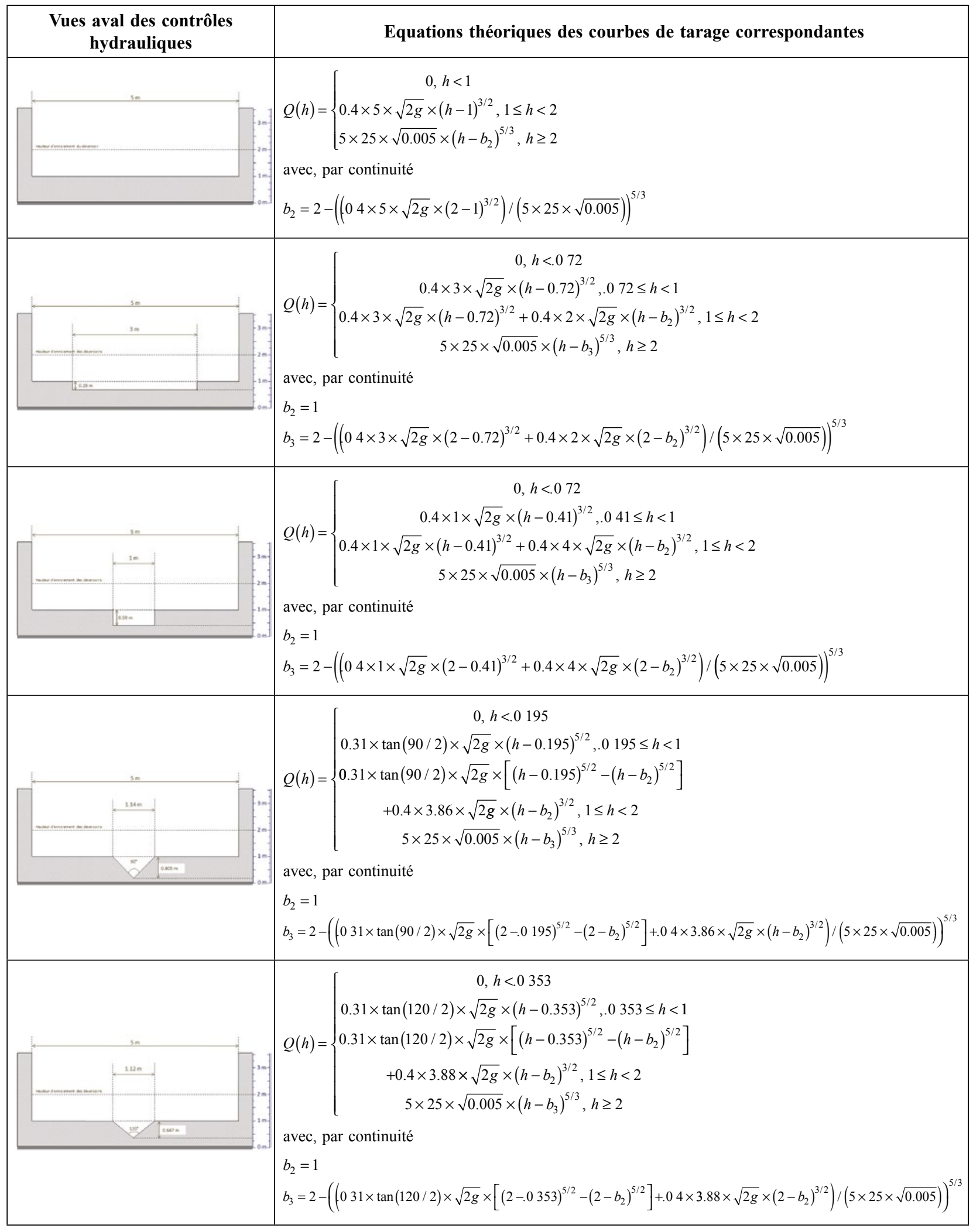


Annexe 2. Valeurs a priori des paramètres des courbes de tarage des cinq stations fictives utilisées pour l'estimation des courbes de tarage et des hydrogrammes avec BaRatin.

\begin{tabular}{|c|c|c|c|}
\hline Contrôle & $\boldsymbol{k}_{k}$ & $a_{k}$ & $c_{k}$ \\
\hline \multicolumn{4}{|c|}{ Cas 0 : Déversoir rectangulaire sans encoche } \\
\hline Déversoir $(k=1)$ & $1 \pm 0.05$ & $8.86 \pm 2$ & $1.5 \pm 0.05$ \\
\hline Chenal $(k=2)$ & $2 \pm 0.2$ & $8.84 \pm 2$ & $1.67 \pm 0.05$ \\
\hline \multicolumn{4}{|c|}{ Cas 1 : Encoche rectangulaire $(L=3 m)$} \\
\hline Déversoir $(k=1)$ & $0.72 \pm 0.05$ & $5.31 \pm 1.5$ & $1.5 \pm 0.05$ \\
\hline Déversoir $(k=2)$ & $1 \pm 0.05$ & $3.54 \pm 1$ & $1.5 \pm 0.05$ \\
\hline Chenal $(k=3)$ & $2 \pm 0.2$ & $8.84 \pm 2$ & $1.67 \pm 0.05$ \\
\hline \multicolumn{4}{|c|}{ Cas 2 : Encoche rectangulaire $(\mathrm{L}=1 \mathrm{~m})$} \\
\hline Déversoir $(k=1)$ & $0.41 \pm 0.05$ & $1.77 \pm 0.5$ & $1.5 \pm 0.05$ \\
\hline Déversoir $(k=2)$ & $1 \pm 0.05$ & $7.09 \pm 2$ & $1.5 \pm 0.05$ \\
\hline Chenal $(k=3)$ & $2 \pm 0.2$ & $8.84 \pm 2$ & $1.67 \pm 0.05$ \\
\hline \multicolumn{4}{|c|}{ Cas 3 : Encoche triangulaire $\left(\right.$ Alpha $\left.=90^{\circ}\right)$} \\
\hline Déversoir $(k=1)$ & $0.195 \pm 0.05$ & $1.57 \pm 0.5$ & $2.5 \pm 0.05$ \\
\hline Déversoir $(k=2)$ & $1 \pm 0.05$ & $6.84 \pm 1.5$ & $1.5 \pm 0.05$ \\
\hline Chenal $(k=3)$ & $2 \pm 0.2$ & $8.84 \pm 2$ & $1.67 \pm 0.05$ \\
\hline \multicolumn{4}{|c|}{ Cas $4:$ Encoche triangulaire $\left(\right.$ Alpha $\left.=120^{\circ}\right)$} \\
\hline Déversoir $(k=1)$ & $0.353 \pm 0.05$ & $2.38 \pm 0.5$ & $2.5 \pm 0.05$ \\
\hline Déversoir $(k=2)$ & $1 \pm 0.05$ & $6.88 \pm 1.5$ & $1.5 \pm 0.05$ \\
\hline Chenal $(k=3)$ & $2 \pm 0.2$ & $8.84 \pm 2$ & $1.67 \pm 0.05$ \\
\hline
\end{tabular}

